\title{
Efecto de la Motivación sobre la Profundidad en los Procesos de Estudio en Universitarios de Formación en Pedagogía
}

\author{
Effect of Motivation on the In-depth Study Processes Among \\ Student Teachers
}

\author{
Jorge A. Salgado * \\ Francisco José Leria \\ María E. Pilar Franco \\ Ximena R. Gajardo \\ María Verónica Olivares \\ Universidad de Atacama
}

\begin{abstract}
El objetivo de la investigación es analizar la asociación entre la motivación y sus perfiles con los enfoques de aprendizaje (superficial y profundo) y variables sociodemográficas. Participaron 201 estudiantes de una universidad pública, pertenecientes a cuatro carreras de formación en Pedagogía que cumplimentaron las escalas de Motivación Académica (EMA), de Procesos de Estudio (R-CPE-2F); y una encuesta ad doc. Se utilizó una estrategia asociativa-comparativa transversal. Los resultados indicaron la existencia de relaciones positivas entre la Amotivación con los procesos de estudio superficiales. Se encontraron diferencias en función del sexo en la Amotivación, Regulación Externa e Identificada, y en el Enfoque y Estrategia Superficial, siendo las medias más altas en los hombres. Se determinaron dos perfiles motivacionales, uno con puntuaciones bajas en motivación intrínseca y extrínseca, y el segundo con puntuaciones altas en Motivación al Logro y Regulación Introyectada. También, diferencias en el Enfoque de Aprendizaje Profundo, siendo las medias más altas en el Clúster 2. Los análisis de regresión múltiple mostraron que la Motivación Intrínseca (MI), las Experiencias Estimulantes y el Logro predicen el $41 \%$ de la variabilidad en el Enfoque Profundo; y la Amotivación y MI al Conocimiento predicen el 28\% para el Enfoque Superficial.
\end{abstract}

Descriptores: Motivación, Formación, Docente, Universidad, Educación.

The objective of the research is to analyze the association between motivation and motivation's profiles with the learning approaches (superficial and deep) and some sociodemographic variables. Participated 201 students from a public university, belonging to four studies in Pedagogy that completed the Scale of Academic Motivation (EMA), Study Processes (R-CPE-2F); and a sociodemographic survey ad doc. A cross-sectional associative-comparative strategy was used. The results indicated the existence of positive relations between the Amotivation and the superficial study processes. Differences were found according to sex in the Amotivation, External and Identified Regulation, and in the Approach and Superficial Strategy, being the highest means in men. Two motivational profiles were determined, one with low scores on intrinsic and extrinsic motivation, and the second with high scores on Achievement Motivation and Introjected Regulation. Differences were found in the Deep Learning Approach, the highest means being in the Cluster 2. Multiple regression analyzes showed that Intrinsic Motivation (MI) to Stimulating Experiences and Achievement, predict $41 \%$ of the variability in the Deep Approach; and Amotivation and MI to Knowledge predict 28\% for Superficial Approach.

Keywords: Motivation, Training, Teachers, Universities, Education.

*Contacto: jorge.salgado@uda.cl

ISSN: 1696-4713

www.rinace.net/reice/

revistas.uam.es/reice
Recibido: $\quad 3$ de agosto 2017

$1^{\text {a }}$ Evaluación: 31 de agosto 2017

$2^{\text {a }}$ Evaluación: 8 de septiembre 2017

Aceptado: 12 de septiembre 2017 


\section{Introducción}

En el contexto general de la diagnosticada crisis en la educación chilena (Montecinos, 2014; Pedraja-Rejas y Rodríguez-Ponce, 2015), se ha planteado una serie de inquietudes por la mejora constante de la formación de los y las estudiantes de pedagogía. Tema presente en el debate académico por más de dos décadas, ha adquirido cada vez una mayor importancia el dominio de un conjunto de saberes pedagógicos y competencias terminales necesarias para el ejercicio de una actividad profesional de calidad (Leguizamon, 2014). Por ejemplo, se ha visualizado la necesidad de incurrir en medidas dirigidas al desarrollo de competencias profesionales orientadas a fortalecer lo que ocurre en el microcosmos de cada escuela (Raczynski et al., 2013); específicamente, en la capacidad del futuro profesional de promover ambientes centrados en el aprendizaje, de los cuales -por ejemplo-, la evidencia empírica señala que poseen un importante impacto en el clima escolar motivacional (Leal-Soto, Ramírez y Valdivia, 2014); en los estilos y profundidad de estudio (Caballero, Norambuena, Gálvez y Salam, 2015); y finalmente, en el aprendizaje y éxito académico (Martínez-Garrido y Murillo, 2016).

En el contexto por una formación inicial docente de calidad, las universidades agrupadas en el Consejo de Rectores de Chile a partir del año 2008 comenzaron con la aplicación de una medición estandarizada conocida como la Prueba Inicia, con el fin de monitorear el nivel de conocimientos (saberes pedagógicos y disciplinares) de los egresados de educación parvularia y pedagogía básica (MINEDUC, 2005). Los resultados obtenidos mostraron que menos de un tercio alcanzó niveles de logro superiores al 75\% (Educación 2020, 2014). Estos bajos estándares de excelencia generaron la necesidad de proponer las modificaciones curriculares necesarias para garantizar una mayor calidad del proceso formativo docente, dando pie a la elaboración de diversas propuestas para su mejoramiento (Cisternas, 2011; Santelices et al., 2015).

Han sido variadas las estrategias propuestas por las instituciones públicas y de educación superior para la mejora progresiva de la formación inicial de los futuros docentes. Por ejemplo, se han ejecutado políticas de acreditación externa especializada (Martínez, Tobón y Sandoval, 2017), a través del ejercicio de la Ley de Aseguramiento de la Calidad de la Educación Superior (Gobierno de Chile, 2006); se ha promovido la generación de estándares orientadores de la formación en pedagogía (MINEDUC, 2011); y/o se han acordado puntajes mínimos de ingreso a las carreras de pedagogía (Ávalos, 2014). A la par, los modelos y enfoques educativos han dado una respuesta a estas contingencias, experimentando una evolución teórica que ha transformado la noción del estudiante a una más activa y responsable de la conducción de sus procesos de aprendizaje (Loyens y Rikers, 2011); previendo con ello el desarrollo de una actitud más activa y diligente con el desarrollo de las competencias necesarias para un buen ejercicio profesional. Como política educativa, esta transformación se hizo palpable con la aceptación de los lineamentos del Proyecto Tuning (Tuning, 2007), el cual busca implementar los principios de Bolonia en el contexto latinoamericano $-\mathrm{y}$ entre otros elementos- el Sistema Europeo de Transferencia de Créditos. Este sistema se adoptó a partir de las iniciativas del Programa de mejoramiento de la calidad y la equidad en la educación terciaria y el Consejo de Rectores de las Universidades Chilenas (CRUCH, 2013); que iniciaron su implementación en los procesos de rediseño curricular con el objetivo de mejorar la legibilidad de los programas de estudio, conocer la demanda real del trabajo académico, equilibrar la carga académica y promover la movilidad estudiantil (Cisterna, Soto y Rojas, 2016). 
La implementación del rediseño curricular estableció entonces una relación directa entre las competencias, los resultados de aprendizaje y la dedicación del estudiante para el cumplimiento de las actividades planificadas (Dochy y Nickmans, 2005, como se citó en Cisterna, Soto y Rojas, 2016); convirtiéndose en una variable relevante, el tiempo y calidad de la dedicación autónoma que un estudiante le entrega al estudio. Si bien la promoción de estas políticas educativas ha sido objeto de importantes visiones criticas (Espinoza, 2014), su aplicación ha promovido la investigación respecto de las variables propias del sujeto que inciden en la profundidad y calidad del estudio, incluyendo el tiempo de dedicación autónoma al aprendizaje, el desempeño académico y satisfacción con el proceso formativo (Cejudo, López y Rubio, 2016). Como resultado se ha promovido la incorporación de las denominadas metodologías activas en el aprendizaje (Rodríguez, Ramírez y Fernández, 2017). Estas metodologías han enfatizado una visión de la formación universitaria más allá de la mera transmisión del conocimiento, sino que buscan potenciar las capacidades profundas de aprendizaje del estudiante, desarrollo de sus habilidades, competencias y/o actitudes sociales, o según Fernández (2006), pretende en el estudiante promover: "aprender a aprender y a desarrollar la curiosidad, aprender a prever y a hacer frente a problemas nuevos, a analizarlos sistemáticamente y a idear soluciones alternativas; aprender a sacar conclusiones a través de fuentes diversas, y aprender a relacionar los conocimientos con la realidad" (p. 314). Los procesos de estudio y las variables que le impactan son importantes en la aplicación de las metodologías activas, destacando el rendimiento académico; las estrategias y uso de las metodologías activas en los docentes; uso de las TICS; precisión de los perfiles estudiantiles de aproximación al estudio; y evaluación de los aprendizajes basada en competencias (Gros, 2007).

Existe una amplia tradición investigativa de más de 40 años en relación a la profundidad en los procesos de estudio, enfoques y estilos de aprendizaje. Se ha mostrado que los estudiantes poseen variados tipos de personalidad, ritmos, estrategias y estilos de abordar el estudio, con impacto en la forma en que aprenden, los profesores enseñan y como ambos interactúan en la relación de enseñanza-aprendizaje (Luzio, Araneda, Salgado y Rain, 2015). Hoy en la ciencia pedagógica se acuña ampliamente el concepto de profundidad en el estudio, principalmente por su valor teórico y metodológico, pues permite el perfeccionamiento de la práctica pedagógica transversal a todos los niveles educativos. Existen diferentes enfoques en el estudio de la profundidad de los procesos de estudio y en este trabajo se utiliza el enfoque personológico (Campos y González, 2015), el cual asume que las diferencias para aprender: "dependen de las particularidades en los fenómenos estructurales y funcionales de la personalidad, que conforman los dos subsistemas de regulación de esta" (p. 18). Desde sus trabajos iniciales por el grupo de Gotemburgo (Marton y Saljo, 1997), se realizó la distinción esencial entre los estilos profundos y superficiales de aprendizaje y su investigación ha abarcado variables con injerencia en la profundidad de los procesos de estudio con respecto de: sexo (Luengo y González, 2005); autoestima y el rendimiento académico (Fernández, Martínez y Melipillán, 2009); niveles de comprensión del contenido, procesamiento y efecto en el rendimiento cognitivo (Richardson, Abraham y Bond, 2012); percepción del entorno de aprendizaje (Entwistle, McCune y Hounsell, 2003); habilidades cognitivas de resolución de problemas y su impacto en la profundidad del estudio (Gijbels et al., 2005); creencias epistemológicas, estilos de aprendizaje y pensamiento reflexivo (Phan, 2008); estrategias de estudio asociadas a la capacidad de organización del aprendizaje (Entwistle y McCune 
2013); la relación entre los estilos de aprendizaje y el estilo instruccional (Baeten et al., 2015); los resultados de aprendizaje y/o el grado de identificación o interés con el material de estudio (Herrmann, Bager y McCune, 2016). No obstante, sigue siendo el rendimiento académico y la profundidad en los procesos de estudios la variable de estudio de mayor significación y protagonismo en la investigación actual (Villamizar y Romero, 2011).

La definición conceptual de la profundidad en los procesos de estudio desde este enfoque, de acuerdo a Velasco (como se citó en Pantoja, Duque y Correa, 2013), comprende el conjunto de características biológicas, sociales, motivacionales y ambientales que un individuo desarrolla a partir de una información nueva o difícil; para percibirla y procesarla, retenerla y acumularla, construir conceptos, categorías y solucionar problemas, que en su conjunto establecen sus preferencias de aprendizaje y definen su potencial cognitivo. Al hablar de estilos y/o enfoques en la profundidad de estudio, Biggs (1993), plantea dos tipos básicos: el profundo y el superficial, que estarían compuestos en su génesis de elementos situacionales y personales. En la actualidad el uso de esta distinción se mantiene (Dinsmore y Alexander, 2012); agregando el estilo estratégico, caracterizado por la consciencia y capacidad organizativa (Entwistle, McCune y Hounsell, 2003).

El estilo de estudio profundo se caracteriza por estar concentrado en el tema del discurso, asociado a un enfoque activo del aprendizaje y al deseo de comprender el punto principal del contenido, establecer conexiones y extraer conclusiones, o según Pinchao (2016):

El estudiante personaliza la tarea, extrayendo su significado para su proyecto de vida
personal y para la vida diaria; relaciona los contenidos de la tarea con otros
conocimientos y con situaciones cotidianas; procura lograr que el aprendizaje tenga
significación personal. (p. 63)

En términos generales, desde un enfoque profundo, el estudiante se centra en el significado, en su comprensión, se interesa por la tarea y valora el esfuerzo; mientras que, desde un enfoque superficial, el estudiante está centrado en el propio discurso, pasividad y minimización de la tarea, con el fin de reproducir los contenidos estudiados. Considera la tarea como una imposición para la obtención de una calificación, predomina la motivación extrínseca, favorece el aprendizaje mecánico, memorístico y repetitivo; y como resultado consigue un nivel de comprensión superficial y generalmente un bajo rendimiento académico, miedo al fracaso y tendencia a la deserción (Biggs, 1993). La evidencia concluye que estudiantes universitarios que utilizan estrategias más complejas y profundas de estudio, presentan mejor rendimiento académico, y menos reprobaciones que el grupo de estudiantes que utiliza estrategias de aprendizaje más simples y superficiales (Fernández, Martínez y Melipillan, 2009), resultados que coincide con los aportados por Pinchao (2016) o Struyven y sus colaboradores (2006). Estos resultados dejan en evidencia la necesidad de desarrollar un proceso de enseñanza-aprendizaje, que tome en consideración las diferencias individuales de los estudiantes para la potenciación de su proceso de estudio, que finalmente influya exitosamente en una capacidad sostenida de logro académico (Cañizares y Guillén, 2013).

En concordancia con las tendencias declaradas en educación de: "contribuir al desarrollo global de cada persona: cuerpo y mente, inteligencia, sensibilidad, sentido estético, responsabilidad individual, espiritualidad" (Delors, 1996, p. 99); se empezó a incluir en el currículo el desarrollo de las denominadas competencias genéricas y/o transversales, las cuales abren un espacio explícito para el desarrollo de capacidades y habilidades blandas, que impacta en la calidad de los procesos de estudio y finalmente la calidad de la formación 
universitaria y desempeño profesional. Su inclusión es parte de una de las tendencias fundamentales en la construcción del currículo actual, cada vez más reconocida para la educación en el siglo XXI, a veces denominado: el aprender a vivir (Tedesco, 2011). Además, el estudio de los estilos y profundidad en los procesos de estudio ha ido en línea con una tendencia mayor de devolver la variable humana al proceso de aprender (Bisquerra y Núria, 2012); y con ello incluir no solamente las variables de adquisición conceptual, sino todas aquellas que posean una incidencia en el aprendizaje, como los aspectos motivacionales y emocionales (Moreno y Torregrosa, 2015); con el consecuente diseño de metodologías y evaluaciones que influyan directa y significativamente en la forma de aprender de los estudiantes y su calidad formativa, disminuyendo las tasas de deserción y aumentando la retención (Gargallo, 2009).

La motivación y el desempeño universitario han sido variables ampliamente estudiadas en educación (Cornejo y Redondo, 2007; Maquilón y Hernández, 2011; Valenzuela et al., 2015). Los estudios muestran que la motivación está relacionada con la estrategia de aprendizaje que utiliza el estudiante para el cumplimiento de sus metas académicas (Coll, Palacios y Marchesi, 2005). Teóricamente se ha afirmado incluso que la interacción motivo-estrategia es lo que define más apropiadamente al concepto de estilo y/o enfoque de aprendizaje (Biggs, 1985, como se citó en Moreno y Torregrosa, 2015); y es lo que lleva al estudiante a ser más consciente de sus motivos y a elegir la estrategia más adecuada a la tarea requerida, generándose consecuentemente una estrecha relación entre el enfoque de aprendizaje y su perfil motivacional. La evidencia sugiere que la motivación intrínseca es aquel proceso psicológico que tiene una mayor implicación en el enfoque de estudio profundo; y la motivación extrínseca es más propia del estilo superficial de estudio (Doménech y Gómez, 2011). Por lo tanto, es posible afirmar que los estudiantes con motivación intrínseca estarán más comprometidos en su proceso de aprendizaje, obtendrán mejores resultados académicos.

Por otra parte, la amplia evidencia existente en torno a la relación entre el componente motivacional del comportamiento y la vivencia emocional; sugiere otra importante línea de investigación dirigida al impacto de las emociones en la motivación, aprendizaje y consecuentemente en la profundidad en los procesos de estudio. Hoy existe un creciente asentimiento respecto al papel de la dimensión socioemocional en educación como gestores de los aprendizajes y buena convivencia (Durlak et al., 2011); y las evidencias se muestran a favor de su desarrollo (Schutte, Malouff y Thorsteinsson, 2013). Según algunos autores (Pentaraki y Burkholder, 2017), la psicología educacional no ha direccionado adecuadamente la evidencia respecto del papel de las emociones en el rendimiento académico, a diferencia de otras disciplinas científicas que le han prestado una atención más explícita, como por ejemplo la neurociencia (Puebla y Talma, 2011). Otros autores, De la Fuente y sus colaboradores (2016), plantean las experiencias emocionales positivas en el aprendizaje pueden facilitar al estudiante el logro de sus metas académicas y solucionar los problemas relacionados al estudio creativamente; por ejemplo, promover su desempeño académico (Ferragut y Fierro, 2012), facilitar el trabajo bajo presión (Laborde et al., 2014), utilizar estrategias efectivas de autorregulación conductual para el estudio (Gugliandolo y Costa, 2014) y/o contribuir con una sensación subjetiva de felicidad (Kanazawa, 2014). Por otra parte, las emociones negativas pueden interferir directamente en los logros académicos (Frank et al., 2014).

La institución de educación superior en la cual se llevó a cabo este estudio declara en su modelo educativo de 2017 la formación de nuevos profesionales desde un enfoque socio- 
constructivista del aprendizaje, lo que implica que el estudiante que aprende es el centro del proceso. El conocimiento lo construye articulando y relacionando lo previo y lo nuevo, de manera significativa y útil en la práctica cotidiana para la resolución de problemas. Además, busca proyectar un espacio que propicie la formación integral y que se distingue, por una parte, en una formación propia, auténtica, con sello y a su vez, con las demandas sociales y en un marco de convergencia con otras entidades de educación superior (UDA, 2012). El Modelo Educativo Universidad de Atacama es por definición un ambiente educativo centrado en el estudiante (Asún, Zúñiga y Ayala, 2013); se requiere por parte del mismo del aprendizaje activo y significativo, a través de una actitud, comportamiento e interpretación activa y profunda de la información de estudio seleccionada (Renkl, 2009). Los modelos centrados en el estudiante declaran la importancia de promover la adopción de estilos de aprendizaje significativo y estudio profundos, con el propósito de lograr congruencia con las demandas que por definición exigen este tipo de ambientes educativos (Struyven et al., 2006); en pos del subsecuente éxito académico esperado.

De esta manera, se plantea como problema de estudio analizar la asociación entre perfil motivacional y profundidad en los procesos de estudio en universitarios de una institución de educación superior durante el proceso inicial de la implementación de su modelo educativo centrado en el estudiante.

\section{Método}

Diseño

El estudio corresponde a una investigación empírica, con una estrategia asociativa de tipo comparativa transversal y un diseño de grupos naturales. No se manipulan variables y se analizan las relaciones de estas indagando las diferencias entre dos o más grupos de individuos a partir de los contrastes generados por la naturaleza y la sociedad (Ato, López y Benavente, 2013).

\section{Participantes}

El muestreo utilizado es de tipo intencionado o por conveniencia. Participaron 202 estudiantes, de una universidad pública, pertenecientes a cuatro carreras de formación en Pedagogía. Se descartó del análisis un participante por no cumplimentar todos los instrumentos, de manera que la población diana se redujo a 201 estudiantes (160 mujeres y 41 hombres), con edades comprendidas entre los 18 y 49 años $(M=21,75 D E=3,949)$, de los cuales el 42,1\% cursaba primer nivel, el 19,3\% el segundo nivel, el 21,8\% el tercer nivel, y el $13,4 \%$ el cuarto nivel y el $3,5 \%$ el quinto nivel. Respecto del estado civil, el $95,5 \%$ de los estudiantes estaban solteros y el 3,5\% casados. Además, el 20,8\% informo tener hijos, y el $78,2 \%$ no tenerlos.

\section{Instrumentos}

Los participantes del estudio completaron tres instrumentos diferentes de recogida de información:

Escala de Motivación Académica-EMA- (Núñez, Martí y Navarro, 2005). El instrumento está formado por 28 reactivos, que comienzan con la frase “¿Por qué estudias?” y se distribuyen en siete subescalas que son: Desmotivación (ej. "No sé por qué voy al instituto y, sinceramente, no me importa"), Regulación Externa (ej. "Para poder conseguir, posteriormente, un mejor salario"), Regulación Introyectada (ej. "Porque cuando hago 
bien las tareas en clase me siento importante"), Regulación Identificada (ej. "Porque me ayudará a tomar una mejor decisión en lo que respecta a mi orientación profesional”), Motivación Intrínseca al Conocimiento (ej. "Porque mi estudios me permiten seguir aprendiendo muchas cosas que me interesan”), Motivación Intrínseca al Logro (ej. "Por la satisfacción que siento cuando voy superando actividades académicas difíciles") y Motivación Intrínseca a las Experiencias Estimulantes (ej. "Porque realmente me gusta asistir a clase"). Las respuestas están graduadas de acuerdo a una escala Likert de siete puntos ( $1=$ no se corresponde en absoluto a $7=$ se corresponde totalmente) (Moreno y Torregrosa, 2015).

Cuestionario de Procesos de Estudio-R-CPE-2F- (Recio y Cabrero, 2005). Instrumento compuesto por 20 ítems con dos categorías de enfoques de aprendizaje: profundo (DA) y superficial (SA) y cuatro subescalas: Motivación Profunda (DM), Estrategia Profunda (DS), Motivación Superficial (SM) y Estrategia Superficial (SS). Corresponde a una escala tipo Likert graduada en cinco puntos $(1=$ nunca o casi nunca es verdadero para mí a $5=$ siempre o la mayoría de las veces es verdadero para mi).

Breve Encuesta Sociodemográfica. Fue elaborada ad hoc para levantar información referente a variables cuantitativas y cualitativas de tipo sociodemográfico y relacionadas con la carrera que estudian, nivel cursado, asignaturas reprobadas, entre otras.

\section{Procedimiento}

Este trabajo fue revisado y aprobado por un Comité de Idoneidad Científica de la institución de los investigadores y contó con la autorización de los directivos de la universidad participante, y los pasos a seguir fueron: (1) Se comunicó de los objetivos del estudio a los a los directores de las carreras colaboradoras. (2) Coordinación con Directores para determinación del lugar de aplicación de los instrumentos. (3) Se informó a los participantes de la finalidad de la investigación y del resguardo de la confidencialidad de la información, además se recalcó el carácter voluntario y la posibilidad de interrumpir la participación en el proceso. (4) Se procedió a la lectura del consentimiento informado y posteriormente a la aplicación anónima de los instrumentos. Los estudiantes no recibieron ningún incentivo por participar, y se tomaron todos los resguardos para garantizar el cumplimiento de los aspectos éticos de la investigación con seres humanos.

\section{Análisis de datos}

Para describir las dimensiones del EMA y el R-CPE-2F, se calcularon sus promedios y desviaciones estándar, así como las correlaciones lineales de Pearson $(r)$ entre las puntuaciones directas obtenidas con ambos instrumentos. Luego se realizó un análisis de las diferencias en la motivación académica y los enfoques de aprendizaje en cuanto al sexo utilizando la prueba de $t$ de Student, y dos MANOva para el nivel y carrera cursada. A continuación, se realizó un análisis de conglomerados (Breckenridge, 2000), con el fin de encontrar grupos de estudiantes con perfiles motivacionales afines. Posteriormente se utilizó la prueba de Chi-cuadrado de Pearson, para examinar la distribución de los perfiles encontrados en función del sexo, carrera y nivel cursado. Seguidamente, para comprobar la existencia de diferencias significativas en los perfiles en función de los procesos de estudio se utilizó la prueba de $t$ de Student para muestras independientes. En el caso de no existir homogeneidad, se aplicó el test de Welch (Armitage, Berry y Matthews, 2001). Para determinar el Tamaño del Efecto (TE) se calculó la $d$ de Cohen $(d)$ y el Eta Cuadrado Parcial $\left(\eta_{P}^{2}\right)$ (Cohen, 1988). Finalmente, se ingresó a un modelo de regresión lineal 
múltiple, con el método por pasos (stepwise), para examinar la capacidad predictiva de las dimensiones de la Escala de Motivación Académica en conjunto con algunas variables sociodemográficas sobre la profundidad o superficialidad en los procesos de estudio. Para el procesamiento de los datos se utilizó el software SPSS 22.0.

\section{Resultados}

\subsection{Análisis descriptivo y correlacional}

En el cuadro 1 aparecen los estadísticos de las subescalas que componen la R-CPE-2F y EMA. En líneas generales, la DA y SA presentan valores elevados en sus medias, siendo mayor en la primera. En cambio, la SM y SS obtuvieron valores más bajos. Con respecto a la motivación académica, la RID, MIC y MIL presentaron valores elevados, mientras que la Amotivación mostró un valor bajo en su media. En cuanto a las correlaciones, el cuadro 1 muestra como el Enfoque Profundo, el Motivo Profundo y la Estrategia Profunda mantienen una relación positiva y significativa con el RID, MIC, MIL y MIEE. Por otra parte, la Amotivación se asoció positivamente con el Enfoque Superficial, el Motivo Superficial y la Estrategia Superficial. La Motivación Intrínseca al Conocimiento se relacionó de forma negativa con el Enfoque Superficial y el Motivo Superficial, siendo más significativo con el segundo.

Cuadro 1. Estadísticos descriptivos y análisis correlacional

\begin{tabular}{|c|c|c|c|c|c|c|c|c|}
\hline & $M(D S)$ & AMOTIVACIÓN & RE & RI & RID & MIC & MIL & MIEE \\
\hline $\mathrm{DA}$ & $37,01(4,68)$ & $-0,054$ & 0,015 & $0,159^{*}$ & $0,267 * *$ & $0,473^{*} *$ & $0,374 * *$ & 0,560 *** \\
\hline SA & $25,93(6,82)$ & $0,398 * *$ & $0,224^{* *}$ & $0,206^{* *} *$ & 0,015 & $-0,167^{*}$ & $-0,086$ & $-0,042$ \\
\hline $\mathrm{DM}$ & $19,18(2,59)$ &,- 122 & 0,009 & 0,132 & $0,238^{* * *}$ & $0,428 * *$ & $0,340^{* * *}$ & 0,464 *** \\
\hline DS & $17,85(2,75)$ & 0,052 & 0,027 & $0,148^{*}$ & $0,235 * *$ & $0,399^{*} *$ & $0,314^{* *}$ & $0,515^{* *}$ \\
\hline SM & $12,20(3,56)$ & $0,368^{*} *$ & $0,141^{*}$ & $0,155^{*}$ & $-0,022$ & $-0,227$ *** & $-0,107$ & $-0,031$ \\
\hline SS & $13,68(3,84)$ & $0,358 * *$ & $0,270^{* * *}$ & 0,248 *** & 0,062 & $-0,090$ & $-0,028$ & $-0,048$ \\
\hline Amotivación & $7,03(4,94)$ & & & & & & & \\
\hline $\mathrm{RE}$ & $21,91(5,79)$ & & & & & & & \\
\hline RI & $22,06(5,44)$ & & & & & & & \\
\hline RID & $25,18(3,01)$ & & & & & & & \\
\hline MIC & $24,81((3,23)$ & & & & & & & \\
\hline MIL & $24,27(3,69)$ & & & & & & & \\
\hline MIEE & $19,78(4,71)$ & & & & & & & \\
\hline
\end{tabular}

Nota: ${ }^{*} \mathrm{p}<0,05,{ }^{*} \mathrm{p}<0,01$. DA: Enfoque Profundo, SA: Enfoque Superficial, DM: Motivo Profundo, DS: Estrategia Profunda, SM: Motivo Superficial, Ss: Estrategia Superficial, RE: Regulación Externa, RI: Regulación Introyectada, RID: Regulación Identificada, MIC: Motivación Intrínseca al Conocimiento, MIL: Motivación Intrínseca al Logro, MIEE: Motivación Intrínseca a las Experiencias Estimulantes.

Fuente: Elaboración propia.

\subsection{Análisis de diferencias en la motivación académica y los enfoques de aprendizaje}

Respecto de las diferencias en las subescalas de la motivación académica en función del sexo, se encontró medias desiguales en: Amotivación, $t(194)=2,333, p=0,021, d=0,365$, 95\% IC [0,302, 3,603], MI a las Experiencias Estimulantes, $t(186)=2,591, p=0,010, d=$ $0,471,95 \%$ IC $[0,513,3,788]$; Regulación Externa, $t(195)=-2,335, p=0,021, d=0,426$, $95 \%$ IC $[-4,330,-0,366]$; y Regulación Identificada, $t(189)=-2,209, p=0,028, d=0,351$, $95 \%$ IC $[-2,222,-0,125]$, siendo en las puntuaciones medias más altas en los hombres en las dos primeras; y superiores en las mujeres en las dos últimas. En todos los casos TE fue 
pequeño. Respecto de los enfoques de aprendizaje se encontró diferencias significativas en las siguientes dimensiones: Enfoque Superficial, $t(193)=2,190, p=0,030, d=0,396,95 \%$ IC $[0,257,4,912]$; y Estrategia Superficial, $t(195)=2,175, p=0,031, d=0,387,95 \%$ IC $[0,136,2,769]$, siendo las puntuaciones medias más altas en los hombre (TE es pequeño).

Los resultados obtenidos de los efectos principales del nivel cursado sobre las dimensiones de la motivación académica no fueron significativos, Hotelling's $t=0,170 ; \mathrm{F}=0,983, p=$ 0,$491 ; \boldsymbol{\eta}_{p}{ }^{2}=0,041$, y en cuanto a los enfoques de aprendizaje fue significativo, Hotelling's $t$ $=0,179 ; F=2,032, p=0,010 ; \eta_{p}{ }^{2}=0,043$, arrojando el análisis de los efectos intersujetos diferencias significativas en el Enfoque Superficial y la Estrategia Superficial, donde las medias más altas se ubican en los primeros niveles cursados.

Respecto de los efectos principales obtenidos en función a la carrera cursada fueron significativos en cuanto a los enfoques de aprendizaje, Hotelling's $t=0,185 ; F=2,801, p=$ 0,$001 ; \eta_{P}{ }^{2}=0,058$, arrojando además el análisis de los efectos intersujetos la presencia de diferencias significativas en el Enfoque Superficial, el Motivo Superficial, y la Estrategia Superficial. En relación a las dimensiones de la motivación académica no fueron significativos, Hotelling's $t=0,158 ; F=1,220, p=0,229 ; \boldsymbol{\eta}_{p}{ }^{2}=0,050$. En síntesis, TE permite comprobar que el nivel y la carrera cursada provocan un mayor efecto sobre los enfoques de aprendizaje.

\subsection{Análisis de cluster para la obtención de perfiles motivacionales}

Para la obtención de los perfiles motivacionales se utilizó el análisis de conglomerados, considerando la etapa jerárquica y no jerárquica (Hair et al., 1998). En primer lugar, se realizó un análisis de conglomerados jerárquicos a través del modelo de Ward estandarizando todas las variables y usando las puntuaciones Z (Sánchez, Leo, Alonso, Pulido y García, 2015), encontrándose 28 puntuaciones por sobre 3, lo que significa la existencia de outliers o casos atípicos que fueron eliminados del análisis (Moreno y Torregrosa, 2015). En este análisis se incluyó las subescalas de la motivación académica, arrojando el dendograma dos opciones de agrupamiento. Posteriormente se utilizó la técnica no jerárquica de conglomerados K-medias con el fin de confirmar lo arrojado por el análisis jerárquico. El resultado de las dos técnicas utilizadas fue muy consistente, apoyándose la estabilidad de los dos perfiles (cuadro 2).

Cuadro 2. Medias, desviaciones típicas y puntuaciones $\mathrm{Z}$ de las variables en cada clúster

\begin{tabular}{lcccrcr}
\hline & \multicolumn{2}{c}{ CONGLOMERADO $\mathbf{1}(\mathbf{N}=\mathbf{6 3})$} & \multicolumn{2}{c}{ CONGLOMERADO $2(\mathbf{N}=\mathbf{1 1 1})$} \\
\cline { 2 - 7 } & $\boldsymbol{M}$ & $\boldsymbol{D} \boldsymbol{E}$ & $\boldsymbol{Z}$ & \multicolumn{1}{c}{$\boldsymbol{M}$} & $\boldsymbol{D} \boldsymbol{E}$ & $\boldsymbol{Z}$ \\
\hline Amotivación & 7,11 & 4,667 & 0,01735 & 6,68 & 5,165 & $-0,07078$ \\
RE & 18,03 & 5,984 & $-0,67006$ & 24,72 & 3,171 & 0,48439 \\
RI & 17,98 & 4,998 & $-0,74847$ & 24,88 & 2,984 & 0,51968 \\
RID & 23,25 & 3,365 & $-0,64024$ & 26,58 & 1,703 & 0,46293 \\
MIC & 22,30 & 3,206 & $-0,77536$ & 26,30 & 1,787 & 0,46115 \\
MIL & 21,29 & 3,576 & $-0,80815$ & 26,32 & 1,779 & 0,55507 \\
MIEE & 16,68 & 4,329 & $-0,65738$ & 21,77 & 4,085 & 0,42229 \\
\hline
\end{tabular}

Fuente: Elaboración propia.

Como se observa en la figura 1, el primer perfil motivacional, menos autodetermminado, está compuesto por 63 universitarios con puntuaciones ligeramente moderadas en Amotivación y muy bajas en motivación intrínseca y extrínseca. El segundo clúster, más 
autodeterminado, está formado por 111 estudiantes con baja puntuación en Amotivación, moderado en Regulación Externa y altas en Motivación al Logro y Regulación Introyectada.

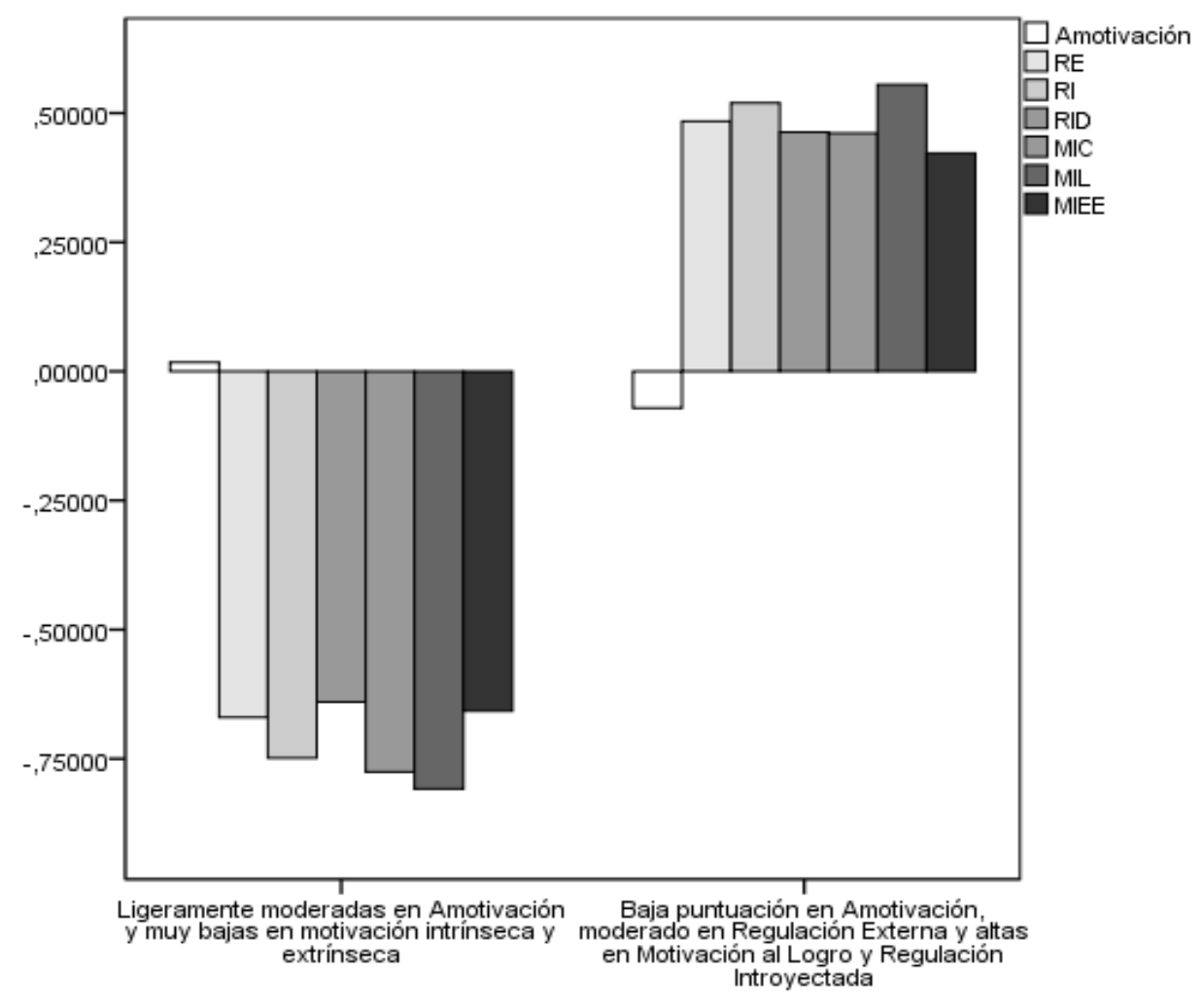

Figura 1. Perfiles motivacionales obtenidos a partir del análisis de clústeres Fuente: Elaboración propia.

\subsection{Análisis de diferencias en los perfiles motivacionales}

En primer lugar, y con el objetivo de comprobar la distribución de los perfiles motivacionales encontrados en función del sexo, nivel y carrera cursada, se utilizó el estadístico Chi-cuadrado de Pearson. En cuanto al sexo, no se presentan diferencias significativas entre hombres y mujeres, $\chi^{2}(1,173)=0,004, p=0,951$. Respecto del nivel cursado, se presenta igualdad de distribución en los perfiles motivacionales, $\chi^{2}(4,173)=$ $9,444, p=0,051$. Por último, no se presentan diferencias en las frecuencias en cuanto al tipo de carrera de pedagogía cursada, $\chi^{2}(3,173)=3,268, p=0,352$.

Por otra parte, como se observa en el cuadro 3, para obtener las diferencias generadas por los perfiles en las estrategias de estudio, se utilizó la prueba $t$ de Student para muestras independientes, encontrándose la presencia de medias desiguales en el Enfoque Profundo, el Motivo Profundo y la Estrategia Profunda, ubicándose las medias más altas en el Clúster 2 (más autodeterminado). Concretamente se ha podido comprobar con el estadístico $d$ de Cohen que los perfiles motivacionales provocan un efecto moderado a mayor sobre el enfoque de aprendizaje profundo. 
Cuadro 3. Diferencias en las estrategias de estudio en función de los perfiles motivacionales

\begin{tabular}{lccccc}
\hline & CLÚSTER 1 & CLÚSTER 2 & \multirow{2}{*}{ T (1 7 1) } & \multirow{2}{*}{$\boldsymbol{P}$} & \multirow{2}{*}{ D DE COHEN } \\
\cline { 2 - 5 } & MEDIA (DE) & MEDIA (DE) & & & \\
\hline Enfoque Profundo (DA) & $34,66(4,808)$ & $38,42(3,870)$ & $-5,566$ & 0,000 & 0,861 \\
Enfoque Superficial (SA) & $25,54(6,048)$ & $26,05(7,295)$ & $-0,466$ & 0,642 & \\
Motivo Profundo (DM) & $18,00(2,776)$ & $19,91(2,178)$ & $-4,989$ & 0,000 & 0,765 \\
Estrategia Profunda (DS) & $16,67(2,682)$ & $18,53(2,537)$ & $-4,549$ & 0,000 & 0,712 \\
Motivo Superficial (SM) & $12,32(3,063)$ & $12,15(3,773)$ & 0,308 & 0,758 & \\
Estrategia Superficial (SS) & $13,22(3,553)$ & $13,87(3,956)$ & $-1,079$ & 0,282 & \\
\hline
\end{tabular}

Fuente: Elaboración propia.

\subsection{Variables predictoras de las estrategias de estudio}

Para explorar la potencia predictiva de las subescalas de la motivación académica, la edad, y el nivel cursado sobre las estrategias de estudio se realizó un análisis de regresión lineal múltiple con el método por pasos (stepwise).

En el cuadro 4 se observan los predictores que entraron en el último paso en la ecuación de regresión para cada estrategia de estudio. Respecto del Enfoque Profundo, fue predicho principalmente por la Motivación Intrínseca (MI) a Experiencias Estimulante y MI al Logro, $F(4,168)=30,177, p<0,001$. En cuanto al Enfoque Superficial, el principal predictor fue Amotivación y MI al Conocimiento, $F(5,165)=14,795, p<0,001$.

Por último, se observa que las varianzas explicadas por las variables predictoras fueron de $41 \%$ para Enfoque Profundo y de 28\% para el Enfoque Superficial.

Cuadro 4. Resumen final del modelo de regresión por pasos en los enfoques de estudio

\begin{tabular}{llcccc}
\hline $\begin{array}{c}\text { ESCALAS DEL } \\
\text { R-CPE-2F }\end{array}$ & V. INDEPENDIENTES & BETA & T & F & $\begin{array}{c}\text { R2 } \\
\text { CoRREGIDA }\end{array}$ \\
\hline \multirow{2}{*}{ Enfoque } & MI a experiencias & 0,406 & $5,358^{* * *}$ & & \\
Profundo & MI al logro & 0,287 & $3,235^{* * * *}$ & $30,177^{* * * *}$ & 0,41 \\
& Regulación introyectada & $-0,209$ & $-2,805^{* *}$ & & \\
& MI al Conocimiento & 0,172 & $2,004^{*}$ & & \\
& Amotivación & 0,312 & $4,715^{* * *}$ & & \\
Enfoque & Edad & $-0,211$ & $-3,254^{* *}$ & & \\
Superficial & Regulación externa & 0,159 & 1,854 & $14,795^{* * * *}$ & \\
& MI al conocimiento & $-0,302$ & $-4,192^{* * *}$ & & \\
& Regulación introyectada & 0,2 & $2,251^{*}$ & & \\
\hline
\end{tabular}

Notas: MI, Motivación Intrínseca. ${ }^{* * *} p<0,001, * * * 0,01, * 0<0,05$.

Fuente: Elaboración propia.

\section{Discusión y conclusiones}

El objetivo del estudio fue analizar la asociación entre la motivación académica y los enfoques de estudio, considerando la relaciones y diferencias entre las dimensiones cognitivas (profundidad en los procesos de estudio) y las dimensiones afectivomotivacionales (perfiles motivacionales).

Se encontraron dos perfiles motivacionales, uno menos autodetermiminado, con puntuaciones ligeramente moderadas en Amotivación y muy bajas en Motivación 
Intrínseca y Extrínseca; y otro más autodeterminado, con una baja puntuación en Amotivación, moderado en Regulación Externa y altas en Motivación al Logro y Regulación Introyectada. Similar a los hallazgos de Moreno y Torregrosa (2015), quienes identifican también dos perfiles, uno con mayores puntuaciones de motivación intrínseca y bajas en desmotivación (más autodeterminado) y otro con mayores niveles de desmotivación, moderados de regulación externa y bajos de motivación intrínseca (menos autodeterminado). Por otra parte, al igual que en el estudio de Romero y colaboradores (2013), se encontraron diferencias estadísticamente significativas en la profundidad de los procesos de estudio en función del sexo, donde las mujeres obtienen más altas puntuaciones en la subescala de enfoque profundo; y por el contrario los hombres, en la subescala del enfoque superficial; hallazgo acorde al periodo del ciclo vital donde se manifiestan las diferencias más marcadas entre sexos (Huang, 2013); y además, acorde a las tendencias observadas en el país, en términos de la superioridad mostradas por las mujeres en varios índices de calidad del proceso formativo (SIES, 2016).

A modo de conclusión, en el análisis de la profundidad de los procesos de estudio en estudiantes universitarios en formación de las carreras de pedagogía, y el diseño simultáneo de estrategias metodológicas por parte del docente en un modelo educativo universitario centrado en el estudiante revela que éste debe incluir tanto los aspectos afectivo-motivacionales como los cognitivos, de los cuales dependerá el éxito en la instauración de los procesos de estudio profundos -y por lo tanto- el desarrollo de la autonomía del estudiante en su aprendizaje y formación universitaria. De esta manera, y considerando los resultados del estudio, es posible visualizar que la motivación intrínseca (experiencias estimulantes al logro y conocimiento), se constituye como el principal predictor del enfoque profundo de estudio, siendo según la literatura y siguiendo a Gargallo y sus colaboradores (2012), el enfoque y/o estilo que favorece con mayor seguridad el éxito académico. Por el contrario, otorgar un mayor valor a lo cognitivo por sobre lo afectivo-motivacional se transformaría en un retroceso.

Además, y a la luz de lo planteado por Baeten y sus colaboradores (2015), para el caso de los estudiantes que no alcanzaron altos resultados en el estilo de estudio profundo la no categorización en este estilo está asociado a la no preferencia por los ambientes de aprendizaje centrados en el estudiante. En línea con la evidencia que sostiene que los estilos de aprendizaje son rasgos cognitivos relativamente estables en el estudiante y no varían significativamente según el entorno (Bolívar y Rojas, 2014). Estos resultados indican una línea de investigación sobre el impacto de la profundidad de los procesos de estudio y el estilo instruccional (ambientes de aprendizaje centrados en el profesor v/s aquellos centrados en el estudiante) (Van Petegem, Donche y Vanhoof, 2005); existiendo evidencias que sugieren la combinación de ambos como un importante motivador para el aprendizaje (Guay, Roy y Valois, 2017).

La percepción de los estudiantes de Pedagogía sobre su formación inicial se ha mostrado variable, y valora los conocimientos prácticos sobre los teóricos (Sotomayor et al., 2013). Esta situación podría tener un efecto desmotivador e iatrogénico en el estilo del estudio profundo y muchas veces de corte más teórico, que no implique la variable pragmática del hacer sobre el saber, temática interesante de proseguir en futuros estudios de esta índole.

Por último, las limitaciones del estudio apuntan al carácter transeccional del diseño, lo que no permite visualizar la progresión en el cambio de un enfoque superficial a uno profundo y su relación temporal con la motivación. No obstante, sus proyecciones se 
perfilan hacia la investigación en los procesos formativos de otras disciplinas y de profesionales en ejercicio, integrando variables como, por ejemplo, el bienestar psicológico y la empatía, con el fin de generar modelos explicativos que incorporen diversos aspectos de la experiencia humana.

\section{Referencias}

Armitage, P., Berry, G. y Matthews, J. (2001). Statistical methods in medical research. Boston, MA: Wiley-Blackwell.

Asún, R., Zúñiga, C. y Ayala, M. C. (2013). La formación por competencias y los estudiantes: confluencias y divergencias en la construcción del docente ideal. Calidad en la Educación, 38, 277-304. https://doi.org/10.4067/S0718-45652013000100008

Ato, M., López, J. y Benavente, A. (2013). Un sistema de clasificación de los diseños de investigación en psicología. Anales de Psicología, 29(3), 1038-1059. https://doi.org/10.6018/analesps.29.3.178511

Ávalos, B. (2014). La formación inicial docente en Chile: Tensiones entre políticas de apoyo y control. Estudios Pedagógicos, 40, 11-28. https://doi.org/10.4067/So71807052014000200002

Baeten, M., Dochy, F., Struyven, K., Parmentier, E. y Vanderbruggen, A. (2015). Student-centred learning environments: An investigation into student teachers' instructional preferences and approaches to learning. Learning Environments Research, 19(1), 443-462. https://doi.org/10.1007/s 10984-015-9190-5

Biggs, J. (1993). What do inventories of students' learning processes really measure? A Theoretical review and clarification. British Journal of Educational Psychology, 63, 3-19.

Bisquerra, R. y Núria, E. (2012). Educación emocional: Estrategias para su puesta en práctica. Adide. Revista de la Asociación de Inspectores de Educación de España, 16, 1-11.

Bolívar, J. M. y Rojas, F. (2014). Estudio de la autopercepción y los estilos de aprendizaje como factores asociados al rendimiento académico en estudiantes universitarios RED. Revista de Educación a Distancia, 44, 60-72.

Breckenridge, J. N. (2000). Validating cluster analysis: Consistent replication and symmetry validating cluster analysis. Multivariate Behavioral Research, 35(2), 261-285. https://doi.org/10.1207/S15327906MBR3502_5

Caballero, M., Norambuena, I., Gálvez, J. L. y Salam, A. M. (2015). Estilos de aprendizaje y rendimiento académico en estudiantes de trabajo social: Un análisis entre México y Chile. Cuadernos de Trabajo Social, 14, 79-100.

Campos, V. y González, I. M. (2015). Sistematización de posiciones teóricas sobre la caracterización de los estilos de aprendizaje. Revista Cubana de Educación Superior, 34(3), 13-28.

Cañizares, Y. y Guillén, A. L. (2013). Auto-conocimiento de los estilos de aprendizaje, aspecto esencial en la actividad de estudio. REICE. Revista Iberoamericana sobre Calidad, Eficacia y Cambio en Educación, 11(3), 123-137.

Cejudo, J., López, M. L. y Rubio, M. J. (2016). Inteligencia emocional y resiliencia: Su influencia en la satisfacción con la vida en estudiantes universitarios. Anuario de Psicología, 46, 51-57. https://doi.org/10.1016/j.anpsic.2016.07.001

Cisternas, T. (2011). La investigación sobre formación docente en Chile: Territorios explorados e inexplorados. Calidad en la Educación, 35, 131-164. https://doi.org/10.4067/S071845652011000200005 
Cisterna, C., Soto, V. y Rojas, C. (2016). Rediseño curricular en la universidad de Concepción: La experiencia de las carreras de formación inicial docente. Calidad en la Educación, 44, $301-$ 323. https://doi.org/10.4067/S0718-45652016000100011

Cohen, J. (1988). Statistical power analysis for the behavioral sciences. Hillsdale, NJ: Lawrence Earlbaum Associates.

Coll, C., Palacios, J. y Marchesi, A. (2005). Desarrollo psicológico y educación. Psicología de la educación escolar. Madrid: Alianza.

CRUCH. (2013). Manual para la implementación de sistema de créditos académicos transferibles. Santiago de Chile: Consejo de Rectores de las Universidades Chilenas.

Cornejo, R. y Redondo, J. M. (2007). Variables y factores asociados al aprendizaje escolar: Una discusión desde la investigación actual. Estudios Pedagógicos, 33(2), 155-175. https://doi.org/10.4067/So7 18-07052007000200009

De la Fuente, J., Martínez, J. M., Salmerón, J. L., Vera, M. M. y Cardelle-Elawar, M. (2016). Actionemotion style, learning approach and coping strategies, in undergraduate university $\begin{array}{llll}\text { students. Anales } & \text { de } & \text { Psicología, } & \text { 457-465. }\end{array}$ https://doi.org/10.6018/analesps.32.2.197991

Delors, J. (1996). Los cuatro pilares de la educación en "La educación encierra un tesoro". Informe a la UNESCO de la Comisión internacional sobre la educación para el siglo XXI. Madrid: Santillana.

Dinsmore, D. y Alexander, P. (2012). A critical discussion of deep and surface processing: What it means, how it is measured, the role of context, and model specification. Educational Psychology Review, 24(4), 499-567. https://doi.org/10.1007/s 10648-012-9198-7

Doménech, F. y Gómez, A. (2011). Relación entre las necesidades psicológicas del estudiante, los enfoques de aprendizaje, las estrategias de evitación y el rendimiento. Electronic Journal of Research in Educational Psychology, 9(2) 463-496.

Durlak, J. A., Weissberg, R. P., Dymnicki, A. B., Taylor, R. D. y Schellinger, K. B. (2011). The impact of enhancing students' social and emotional learning: A meta-analysis of schoolbased universal interventions. Child Development, 82(1), 405-432. https://doi.org/10.1111/j.1467-8624.2010.01564.x

Educación 2020. (2014). Opinión de Educación 2020 sobre resultados de prueba Inicia 2014. Recuperado de http://www.educacion2020.cl/noticia/opinion-de-educacion-2020-sobre-resultados-deprueba-inicia-2014

Entwistle, N. y McCune, V. (2013). The disposition to understand for oneself at university: Integrating learning processes with motivation and metacognition. British Journal of Educational Psychology, 83(2), 267-279. http://doi.org/10.1111/bjep.12010

Entwistle, N., McCune, V. y Hounsell, J. (2003). Investigating ways of enhancing university teaching-learning environments: Measuring students' approaches to studying and perceptions of teaching. En E. De Corte, L. Verschaffel, N. Entwistle, y J. van Merriënboer (Eds.), Powerful learning environments: Unravelling basic components and dimensions (pp. 89107). Oxford: Elsevier Science.

Espinoza, O. (2014). Análisis crítico del discurso de las competencias en la formación inicial docente en Chile. Estudios Pedagógicos, 4O(2), 147-159. https://doi.org/10.4067/So71807052014000300009

Fernández, M. R. (2006). Reseña de "Metodología participativa en la enseñanza universitaria" de Fernando López Noguero. Revista Interuniversitaria de Formación del Profesorado, 20(3), 313316. 
Fernández, O. M., Martínez B. M. y Melipillán, R. (2009). Estrategias de aprendizaje y autoestima: Su relación con la permanencia y deserción universitaria. Estudios Pedagógicos, 35(1), 27-45. https://doi.org/10.4067/S07 18-07052009000100002

Ferragut, M. y Fierro, A. (2012). Inteligencia emocional, bienestar personal y rendimiento académico en preadolescentes. Revista Latinoamericana de Psicología, 44(3), 95-104.

Frank, D., Dewitt, M., Hudgens-Haney, M., Schaeffer, D., Ball, B., Schwarz, N., ...y Sabatinelli, D. (2014). Emotion regulation: Quantitative meta-analysis of functional activation and deactivation. Neuroscience $\mathcal{E}^{2}$ Biobehavioral Reviews, 45, 202-211. https://doi.org/10.1016/j.neubiorev.2014.06.010

Gargallo, B. (2009). Los procesos de enseñanza-aprendizaje en la universidad. Revista Educación y Pedagogía, 19(47), 139-152.

Gargallo, B., Suárez, J. M., García, E., Pérez, C. y Sahuquillo, P. M. (2012). Enfoques de aprendizaje en estudiantes universitarios excelentes y en estudiantes medios. Revista Española de Pedagogía, 70(252), 185-200.

Gijbels, D., Van de Watering, G., Dochy, F. y Van den Bossche, P. (2005). The relationship between students' approaches to learning and the assessment of learning outcomes. European Journal of Psychology of Education, 20, 327-336. https://doi.org/10.1007/BF03 173560

Gobierno de Chile. (2006). Ley de aseguramiento de la calidad de la educación superior. Recuperado de http:/ / leychile.cl/Navegar?idNorma=255323

Gros, B. (2007). Tendencias actuales de la investigación en docencia universitaria. Edusfarm, Revista D’educació Superior en Farmàcia, 1, 1-13.

Guay, F., Roy, A. y Valois, P. (2017). The British psychological society teacher structure as a predictor of students' perceived competence and autonomous motivation: The moderating role of differentiated instruction. British Journal of Educational Psychology, 87(2), 224-240. https://doi.org/10.1111/bjep.12146

Gugliandolo, M. C. y Costa, S. (2014). Trait emotional intelligence as mediator between psychological control and behaviour problems. Journal of Child and Family Studies, 24(8), 2290-2300. https://doi.org/10.1007/s 10826-014-0032-3

Hair, J. F., Anderson, R. E., Tatham, R. L. y Black, W. C. (1998). Multivariate data analysis. Upper Saddle River, NJ: Prentice-Hall.

Herrmann, K. J., Bager, A. y McCune, V. (2016). Investigating the relationships between approaches to learning, learner identities and academic achievement in higher education. High Education, 74(3), 385-400. https://doi.org/10.1007/s 10734-016-9999-6

Huang, C. (2013). Gender differences in academic self-efficacy: A meta-analysis. European Journal of Psychology of Education, 28(1), 1-35. https://doi.org/10.1007/s 102 12-011-0097-y

Kanazawa, S. (2014). Why is intelligence associated with stability of happiness? British Journal of Psychology, 105(3), 316-337. https://doi.org/10.1111/bjop.12039

Laborde, S., Lautenbach, F., Allen, M. S. y Achtze, S. (2014). The role of trait emotional intelligence in emotion regulation and performance under pressure. Personality and Individual Differences, 57, 43-47. https://doi.org/10.1016/j.paid.2013.09.013

Leal-Soto, F., Ramírez, J. D. y Valdivia, Y. (2014). Bienestar psicológico y prácticas docentes con efectos motivacionales orientadas al aprendizaje. Universitas Psycholgica, 13(3), 1037-1046. https://doi.org/10.1 1144/Javeriana.UPSY 13-3.bppd 
Loyens, S. y Rikers, R. (2011). Instruction based on inquiry. En R. Mayer y P. Alexander (Eds.), Handbook of research on learning and instruction (pp. 361-381). Nueva York, NY: Routledge.

Luengo, R. y González, J. J. (2005). Relación entre los estilos de aprendizaje, el rendimiento en matemáticas y la elección de asignaturas optativas en alumnos de ESO. RELIEVE. Revista Electrónica de Investigación y Evaluación Educativa, 11(2), 147-165.

Luzio, A. Q., Araneda, F., Salgado, J. A. y Rain, M. F. (2015). Estilos de aprendizaje de estudiantes y docentes de primer y segundo año de la carrera de medicina veterinaria en Concepción, Chile. Revista de Investigaciones Veterinarias del Perú, 26(4), 725-731. https://doi.org/10.15381/rivep.v26i4.11251

Maquilón, J. J. y Hernández, F. (2011). Influencia de la motivación en el rendimiento académico de los estudiantes de formación profesional. Profesorado, Revista Electrónica Interuniversitaria de Formación del Profesorado, 14(1), 81-100.

Martínez, J., Tobón, S. y Romero, A. (2017). Problemáticas relacionadas con la acreditación de la calidad de la educación superior en América. Innovación Educativa, 17(73), 79-1 16.

Martínez-Garrido, C. y Murillo, F. J. (2016). Investigación Iberoamericana sobre Enseñanza Eficaz. Revista Mexicana de Investigación Educativa, 21(69), 471-499.

Marton, F. y Saljo, R. (1997). Approaches to learning. En F. Marton, D. Hounsell y N. Entwistle (Eds.), The experience of learning (pp. 39-58). Edimburgo: Scottish Academic Press.

MINEDUC. (2005). Informe Comisión Formación Inicial Docente. Santiago de Chile: Serie Bicentenario.

MINEDUC. (2011). Estándares orientadores para egresa - dos de carreras de pedagogía en educación básica. Santiago de Chile: Ministerio de Educación.

Montecinos, C. (2014). Análisis crítico de las medidas de presión propuestas para mejorar la formación inicial de docentes en Chile por el panel de expertos para una educación de calidad. Estudios Pedagógicos, 40, 285-301. https://doi.org/10.4067/So71807052014000200017

Moreno, J. A. y Torregrosa, Y. S. (2015). Perfiles motivacionales de estudiantes universitarios. Procesos de estudio y satisfacción con la vida. Profesorado, Revista Electrónica Interuniversitaria de Formación del Profesorado, 18(3), 169-182. http://doi.org/10.6018/reifop.18.3.200441

Núñez, J., Martín, J. y Navarro, J.G. (2005). Validación de la versión española de la Échelle de motivation en éducation. Psicothema, $17(2), 344-349$.

Pantoja, M. A., Duque, L. I. y Correa, J. S. (2013). Modelos de estilos de aprendizaje: Una actualización para su revisión y análisis. Revista Colombiana de Educación, 64, 79-105.

Pedraja-Rejas, L. y Rodríguez-Ponce, E. (2015). El aseguramiento de la calidad: Un imperativo estratégico en la educación universitaria. Ingeniare. Revista Chilena de Ingeniería, 23(1), 4-5. https://doi.org/10.4067/S07 18-33052015000100001

Pentaraki, A. y Burkholder, G. J. (2017). Emerging evidence regarding the roles of emotional, behavioural, and cognitive aspects of student engagement in the online classroom alexandra. European Journal of Open, Distance and e-Learning, 2O(1), 1-21.

Phan, H. P. (2008). Predicting change in epistemological beliefs, reflective thinking and learning styles: A longitudinal study. British Journal of Educational Psychology, 78(1), 75-93. http://doi.org/10.1348/000709907X204354

Pinchao, L. (2016). Hacia una práctica evaluativa que favorezca el aprendizaje y mejore la enseñanza. Revista UNIMAR, 34(1), 57-69. 
Puebla, R. y Talma, M. P. (2011). Educación y neurociencias. La conexión que hace falta. Estudios Pedagógicos, 37(2), 379-388. https://doi.org/10.4067/S0718-07052011000200023

Raczynski, D., Muñoz, G., Weinstein, J. y Pascual, J. (2013). Subvención escolar preferencial en Chile: Un intento por equilibrar la macro y micro política escolar. REICE. Revista Iberoamericana sobre Calidad, Eficacia y Cambio en Educación, 11(2), 164-193.

Recio, M. A. y Cabrero, J. (2005). Enfoques de aprendizaje, rendimiento académico y satisfacción de los alumnos en formación en entornos virtuales. Revista Píxel-Bit, 25, 93-115.

Renkl, A. (2009). Why constructivists should not talk about constructivist learning environments: A commentary on Loyens and Gijbels (2008). Instructional Science, 37, 495-498. https://doi.org/10.1007/s1 1251-009-9098-5

Richardson, M., Abraham, C. y Bond, R. (2012). Psychological correlates of university students' academic performance: A systematic review and meta-analysis. Psychological Bulletin, 138(2), 353-387. https://doi.org/110.1037/a0026838

Rodríguez, A. B., Ramírez, L. J. y Fernández, W. (2017). Metodologías activas para alcanzar el comprender. Formación Universitaria, 10(1), 79-88. https://doi.org/10.4067/S071850062017000100009

Romero, A., Hidalgo, M. D., González, F., Carrillo, E., Pedraja, M. J., García, J. y Pérez, M. A. (2013). Enfoques de aprendizaje en estudiantes universitarios: Comparación de resultados con los cuestionarios ASSIST y R-SPQ-2F. Revista de Investigación Educativa, 31 (2), $375-$ 391. https://doi.org/10.6018/rie.31.2.151851

Sánchez, D., Leo Marcos, F. M., Alonso, D. A., Pulido-González, J. J. y García-Calvo, T. (2015). Análisis de los perfiles motivacionales y su relación con los comportamientos adaptativos en las clases de educación física. Revista Latinoamericana de Psicología, 47(3), 156-166. https://doi.org/10.1016/j.rlp.2015.06.007

Santelices, M. V., Galleguillos, P., González, J. y Taut, S. (2015). Un estudio sobre la calidad docente en chile: El rol del contexto en donde enseña el profesor y medidas de valor agregado. PSYKHE, 24(1), 1-14. https://doi.org/10.7764/psykhe.23.2.673

Schutte, S., Malouff, J. M. y Thorsteinsson, E. B. (2013). Increasing emotional intelligence through training: Current status and future directions. The International Journal of Emotional Education, 5(1), 56-72. https://doi.org/10.1016/j.neubiorev.2014.06.010

SIES. (2016). Informe brechas de género en educación superior: Datos 2016. Servicio de información de educación superior. Santiago de Chile: SIES.

Sotomayor, C., Coloma, C. J., Parodi, G., Ibáñez, R., Cavada-Hrepich, P. y Gysling-Caselli, J. (2013). Percepción de los estudiantes de pedagogía sobre su formación inicial. Magis, Revista Internacional de Investigación en Educación, 5(1 1), 375-392.

Struyven, K., Dochy, F., Janssens, S. y Gielen, S. (2006). On the dynamics of students' approaches to learning: The effects of the teaching/learning environment. Learning and Instruction, 16(4), 279-294. https://doi.org/10.1016/j.learninstruc.2006.07.001

Tedesco, J. C. (2011). Los desafíos de la educación básica en el siglo XXI. Revista Iberoamericana de Educación, 55, 31-47.

Tuning. (2007). Reflexiones y perspectivas de la Educación Superior en América Latina. Informe final. Bilbao: Publicaciones de la Universidad de Deusto.

UDA. (2012). Modelo educativo. Recuperado de http://www.cmd.uda.cl/ 
Valenzuela, J., Muñoz, C., Silva, I., Gómez, V. y Precht, A. (2015). Motivación escolar: Claves para la formación motivacional de futuros docentes. Estudios Pedagógicos, 41(1), 351-361. https://doi.org/10.4067/S07 18-07052015000100021

Van Petegem, P., Donche, V. y Vanhoof, J. (2005). Relating pre-service teacher's approaches to learning and preferences for constructivist learning environments. Learning Environments Research, 8(3), 309-332. https://doi.org/10.1007/s10984-005-1564-7

Villamizar, G. y Romero, L. (2011). Relación entre variables psicosociales y rendimiento académico en estudiantes de primer semestre de Psicología. Revista de Educación y Desarrollo Social, $5(1), 41-54$.

\section{Breve CV de los autores}

\section{Jorge A. Salgado}

Psicólogo, Doctor en Ciencias de la Educación y Diplomado en Bioética para la Investigación con Seres Humanos. Profesor Asistente y Subdirector del Departamento de Psicología de la Universidad de Atacama. Entre sus intereses académicos destacan: Educación Inclusiva, Salud Mental en contextos organizacionales, y calidad de vida laboral. Atrás áreas de interés son: estrés y factores protectores antes desastres socionaturales, estrés laboral, motivación escolar. Actualmente desarrolla dos proyectos de investigación relacionados con la docencia universitaria y el desarrollo de competencias en universitarios de las distintas facultades académicas. ORCID ID: 0000-0003-24704988. Email: jorge.salgado@uda.cl

\section{Francisco José Lería}

Psicólogo, Magister en Psicología clínica, Post-diplomado en Psicoterapia de Integración. Académico Universitario, profesor asistente (2014-2017); relator empresas (2011-2015); Psicólogo en hogar de menores (2009-2011); psicólogo centro social (2007-2009); psicoterapeuta práctica privada. Entre sus intereses académicos destacan: Educación Contemplativa, Educación emocional, Inclusividad e Integración educativa. Otras áreas de interés son: estrés y factores protectores antes desastres socio-naturales, estrés laboral, motivación escolar. Actualmente está a cargo de un programa para el desarrollo de habilidades contemplativas para estudiantes de educación parvularia en práctica profesional. ORCID ID: 0000-0001-6326-2567. Email: francisco.leria@uda.cl

\section{María E. Pilar Franco}

Es profesora de Estado en Inglés titulada de la Universidad de Santiago de Chile, Licenciada en Educación, postgrado Magíster en Lingüística USACH, Diplomado en Docencia e Investigación Universitaria de la Universidad de Atacama. Pasantías: en "The School of Education at The University of Colorado" at Boulder Colorado, USA, en "Alverno College" Milwaukee, Wiscounsin, USA. Áreas de Experticia: Lingüística Aplicada, Desarrollo de la Competencia Comunicativa en Inglés como Lengua Extranjera (EFL), Lenguaje y Cultura. Académica Departamento de Idiomas, Directora por seis años del mismo departamento, Decana Subrogante Facultad de Humanidades y Educación, actualmente Directora del UDA English Center, Universidad de Atacama. Línea de Investigación en: Docencia Universitaria, Lenguaje y Cultura, Evaluación del aprendizaje. ORCID ID: 0000-0003-0187-0190. Email: pilar.franco@uda.cl 


\section{Ximena R. Gajardo}

Profesora de Estado en Matemáticas, titulada de la Universidad de la Serena, posgrado egresada de Magíster en Informática Educativa de la Universidad Tecnológica Metropolitana, Diplomado en Docencia e Investigación Universitaria de la Universidad de Atacama, Diplomado en Estadística para la Educación de Iplacex. Cursando actualmente postítulo de Mención en Educación Matemática de la Universidad Andrés Bello y postítulo Conducción de Procesos de Formación Docente en Matemática de la Universidad de Chile. Tiene una experiencia Docente de 3 años en pregrado en la Carrera de Pedagogía en Educación General Básica de la Facultad de Humanidades y Educación de la Universidad de Atacama. Experiencia docente de 24 años en el aula en establecimientos Educacionales de Enseñanza media. Actualmente es académica del Departamento de Educación Básica, Facultad de Humanidades y Educación de la Universidad de Atacama. ORCID ID: 0000-0001-8823-6218. Email: ximena.gajardo@uda.cl

\section{$\mathbf{M}^{\mathrm{a}}$ Verónica Olivares}

Profesora de Educación General Básica con Mención en Matemática, titulada de la Universidad de Atacama, posgrado Magíster en Informática Educativa de la Universidad Tecnológica Metropolitana, postítulo en Informática Educativa RPNP 03-1099 de la Universidad de la Frontera, Diplomado en Docencia e Investigación Universitaria de la Universidad de Atacama y Diplomado en Incorporación de Estándares y Competencias TIC en la Formación Inicial de Docentes de la Pontificia Universidad Católica de Valparaíso. Experiencia Docente de 19 años en pregrado en las carreras de pedagogía de la Universidad de Atacama. Experiencia de 15 años como directora de Proyecto "Enlaces" de la Universidad de Atacama, iniciativa del Ministerio de Educación-Chile. Actualmente Directora de Departamento de Educación Básica, Facultad de Humanidades y Educación de la Universidad de Atacama. ORCID ID: 0000-0001-7959-059X. Email: verónica.olivares@uda.cl 